\title{
Obsolescence in Urban Energy Infrastructures: The Influence of Scaling Laws on Consumption Forecasting
}

Ricard Horta-Bernús and Martí Rosas-Casals

ABSTRACT: Cities can be considered complex systems, constantly changing and adapting to new economic, social, and cultural dynamics. They exist in many forms and over a wide range of sizes. In spite of this, researchers have discovered regularities in the form of simple scaling laws that emerge when urban outputs of many types, such as income, patents or energy consumption, are correlated with population size. This article briefly presents some facts and figures on scaling correlations in urban contexts and how this evidence can determine and influence the obsolescence of energy infrastructures. It finally suggests several strategies which could be used to ameliorate the impacts of this performance in urban consumption forecasting assessment processes.

KEYWORDS Complex Systems; Energy Infrastructures; Scaling Laws; Obsolescence; Forecasting

\section{Introduction}

Cities can be considered complex systems (Batty, 2007). From a structural point of view, many parallels have been suggested between cities and other complex systems like ecosystems or biological organisms (Dendrinos and Mullally, 1985; West and Brown, 2005). Complexity is certainly one of the most discussed terms in science. But complex systems do have certain features in common that might be used as a definition. They are usually composed of many independent actors that are constantly interacting and adapting to one another by means of nonlinear feedback loops and most important: they never reach an equilibrium state. Indeed a city is the outcome of many nonlinear dynamic interactions among independent agents: citizens, foreigners, urban landscapes, etc. Buildings, streets, roads, and public spaces

Correspondence Address: Martí Rosas-Casals, SUMM Lab, 1, Colom st., 08222,

Terrassa, Spain. Email: rosas@mmt.upc.edu

(C) 2013 The Society of Urban Technology 
display complex spatial patterns which constantly change due to each city's inherent adaptive nature (Mumford, 1961). When these dynamic processes are taken into account, the spatial and temporal evolution of a city cannot be explained solely by centralized urban planning processes or architectural constraints. Self-organizing processes, with emergent behaviors that cannot be understood only analyzing individual composing elements, are normally the outcome of local interactions among inhabitants, institutions, and urban environments. These are spontaneous, beyond-our-control, and undirected processes that rule the development, growth, and even decay, of every single city.

In light of this reality, will it ever be possible to obtain regularities or similar behavioral patterns among different cities that could help us to understand them analytically? In the last twenty years, compelling techniques of data analysis introduced by researchers from many fields have let to the discovery of surprising characteristics common to all cities, regardless of their individual history and geographical location. Such characteristics involve population and area growth laws (Rozenfeld et al., 2008; Marshall, 2007), fractal growth (Batty and Longley, 1994) and, the oldest known of all, distribution of city sizes that usually follow Zipf's law (Zipf, 1949). In recent years, one particularly interesting statistical pattern which correlates urban infrastructures and socioeconomic activities with cities' size has been observed in some countries (Bettencourt et al., 2007). This statistical relationship, similar to those encountered in the biological realm (Kleiber, 1932), typically follows a simple power law $Y \sim M^{b}$ where $Y$ is some observable, $M$ the mass of the organism and $b$ the scaling exponent. Exponent $b$ has been found to belong to three categories (Bettencourt et al., 2007): (1) $b$ $<1$ (sublinear scaling) associated with infrastructures and services; (2) $b=1$ (linear scaling) associated with clearly defined individual human needs; and (3) $b>1$ (superlinear scaling) associated with outcomes mostly coming from social interactions. Because statistical laws of this kind have been very successful to explain many properties of the biological realm (West et al., 1997), recognizing equivalent laws for urban contexts could be helpful to account for their complexities. As of this writing, a theory that combines the trade-off between the positive feedback associated with social interactions and the negative feedbacks resulting from mixing (i.e., travel and congestion) the population over ever larger areas as the cities get bigger, seems to be a promising one (Bettencourt, 2013).

Be that as it may, the truth is that while sublinear exponents are desirable (i.e., meaning that an increase in size implies economies of scale), superlinear exponents imply increasing returns, like in population growth, very difficult 
to cope with in bounded spatial systems like urban agglomerations (and our planet, by extension). Is in this last category where we can find most of the urban energy consumption outputs like water, fuel or electricity scaling patterns. Thus, as the city grows in terms of inhabitants, energy consumption usually increases faster. And consequently, energy infrastructures could become obsolete sooner than expected.

This paper briefly discusses the effects of superlinear scaling laws in urban energy infrastructures in general and electric networks in particular, and how obsolescence can be moved forward by many years when consumption forecasting processes do not take these laws into account. The first part of this paper examines scaling laws in cities and how these patterns can influence urban development. We then discuss the use of simple tools that allow assessing obsolescence in electricity infrastructures, including discussion of our case study, the autonomous community of Andalusia, the southernmost region of mainland Spain. We finish the paper considering the implications that our study may have upon emerging challenges of cities and a future global energy system.

\section{Scaling in Cities}

Living organisms are amazingly diverse and complex. But nonetheless they share a remarkable and simple characteristic: the scaling behavior of key biological processes over a broad spectrum of phenomena and an immense range of energy and mass. This scaling has been termed allometric, a word that refers to properties in bodies that are not isometric, but that change with size according to a particular law. This observed scaling is typically a simple power law $Y \sim M^{b}$ where $Y$ is some observable, $M$ the mass of the organism and $b$ the scaling exponent. Although an absolute agreement among scholars does not exist and a certain controversy around the precise value of the exponent is under debate, it is shown it approximates a simple multiple of 1/4. In biology, the metabolic rate of living organisms scales as the 3/4-power of mass over 27 orders of magnitude, from molecular and intracellular levels up to the largest organisms. Similarly, time-scales (such as lifespans and growth rates) and sizes (such as bacterial genome lengths, tree heights, and mitochondrial densities) scale with exponents that are typically simple powers of 1/4 (see West et al., 2005 and references therein). Although living organisms are complex systems with hierarchical levels of organization formed by many components and many interactions at different temporal and spatial scales, the simplicity of these models suggest that fundamental 
principles underlay much of the coarse-grained generic structure and organization of living systems. In fact, the observed power law scaling can be explained by means of energy minimization models and space filling constraints (West et al., 1997).

Over the last years much effort has been devoted to developing a unified theory of urban living (Bettencourt and West, 2010). With 52 percent of people (78 percent in more developed regions) now living in cities (United Nations, 2012), sustainability science is in great need of finding a predictive framework where dynamics involved in the on-going expansion processes of urban areas can be included. Per capita indicators (i.e., income, GDP, etc.) assume that, in general terms, some specific characteristics of urban zones increase linearly with size. However, this approach might not be suitable to characterize and to compare cities since it ignores emergent phenomena and nonlinear interactions among composing parts in human agglomerations as a result of their social dynamics (Bettencourt et al. 2010). To take this fact into account, and among many frames of reference (Batty, 2007), scaling appears as a promising one (Bettencourt, 2013). Many authors have shown this statistical trait, correlating urban variables with city size and finding simple power laws like $Y \sim M^{b}$ (Bettencourt et al. 2007, Horta-Bernús et al. 2010, Kühnert et al. 2006, Lämmer et al. 2006). In fact, many properties of cities that characterize their inner dynamics are shown to be power-law functions of population size. In addition, scaling exponents for these properties of cities fall into characteristic classes (Table 1).

\begin{tabular}{llcccc}
\hline Class & \multicolumn{1}{c}{$\mathrm{Y}$} & $\mathrm{b}$ & $95 \%$ CI & $\mathrm{R}^{2}$ & Ref. \\
\hline \multirow{4}{*}{$b>1$} & New patents & 1.27 & {$[1.25,1.29]$} & 0.72 & (b) \\
& Inventors & 1.25 & {$[1.22,1.27]$} & 0.76 & (b) \\
& GDP & 1.15 & {$[1.06,1.23]$} & 0.96 & (b) \\
& Employed in financial intermediation & 1.25 & {$[1.13,1.36]$} & 0.96 & (a) \\
\multirow{3}{*}{$b=1$} & Total employment & 1.01 & {$[0.99,1.02]$} & 0.98 & (b) \\
& Household electricity consumption & 1.04 & {$[0.96,1.12]$} & 0.85 & (c) \\
& All household spaces & 1.00 & {$[0.95,1.16]$} & 1.00 & (a) \\
& Gasoline stations & 0.77 & {$[0.74,0.81]$} & 0.93 & (b) \\
$b<1$ & Length of electrical cables & 0.87 & {$[0.82,0.92]$} & 0.75 & (b) \\
& Road surface & 0.82 & {$[0.71,0.93]$} & 0.93 & (a) \\
\hline
\end{tabular}

Table 1: Selection of scaling exponents and their characteristic classes. Source: Adapted from (a) Arcaute and collaborators (Arcaute et al., 2013), (b) Bettencourt and collaborators (Bettencourt et al., 2007) and (c) Horta-Bernús and collaborators (Horta-Bernús et al., 2010). Note: values from Ref.(a) are only valid for Large Urban Zones (LUZ), a concept which, according to Arcaute and collaborators, might not correspond to a sound definition of city.

(C) 2013 The Society of Urban Technology 
Although universality and robustness of the exponents is under discussion and might depend on a city's morphological and functional definition (see note in Table 1), results seem to indicate that, in contrast with biological systems, where economies of scale associated with optimal and efficient behaviors are the usual outcome, observables of man-made systems like urban agglomerations present more variability. Whereas quantities involved in infrastructural variables also show material economies of scale with sublinear exponents (i.e., $b<1$ ), those reflecting social interactions have superlinear (increasing returns) correlation with population size (i.e., $b>1$ ). This category of scaling exponents implies that as the city grows, its consumption grows more than the value of consumption growth that would have if this was strictly proportional to the increase in population. There is no equivalent to these accelerated growing variables in nature. Given that resources are finite, such growth would lead to a collapse of the system in a finite period of time. In this context, the study of scaling laws for energy variables and infrastructures, when considered as proxies for the basal metabolism rate of the cities, becomes truly significant since it implies a fundamental understanding of the future sustainability of an urban agglomeration.

\section{Scaling and Urban Energy Consumption}

Among all urban observables, those connected with energy consumption in general, and electricity in particular appear to be of paramount importance. Cities can be considered systems of systems (M. Dodgson and D. Gann, 2011). And consequently, also energy systems in urban contexts can be understood as truly complex systems inside complex systems. These can be described in terms of dynamic complex multi-layer structures that integrate hardware, software, users, stakeholders, and policy makers. Inside energy systems, electric power systems in particular are an exemplifying case. Electricity is probably the most used type of energy in the world, representing more than a third of the energy consumed in developed countries (Figure 1). It stands as a fundamental energy vector to industrial and social development, and an essential technological advancement. This type of energy plays an important role in people's lives, as it has allowed many commodities which over the years have become fundamental. Its presence is nowadays so intertwined with ours, and so much taken for granted, that we are only capable of sensing its absence, disguised as a cascading failure or a blackout in its extreme form (Nye, 2010). 
Electric power systems are multi-layer systems characterized by different interconnected and interacting levels, with different scales, both in terms of extension and energy (power) involved: on one hand, high voltage transmission systems which are emerging as global energy infrastructures and spanning over continents; and at a smaller scale, the distribution systems that serve users with local distributed production. As an energy vector, electricity is generally produced by other sources of primary energy, such as fossil fuels. It must also be adapted to adequate voltage values by transformer substations and distributed throughout a region by electric power supply substations and different types of distribution systems (Figure 2).

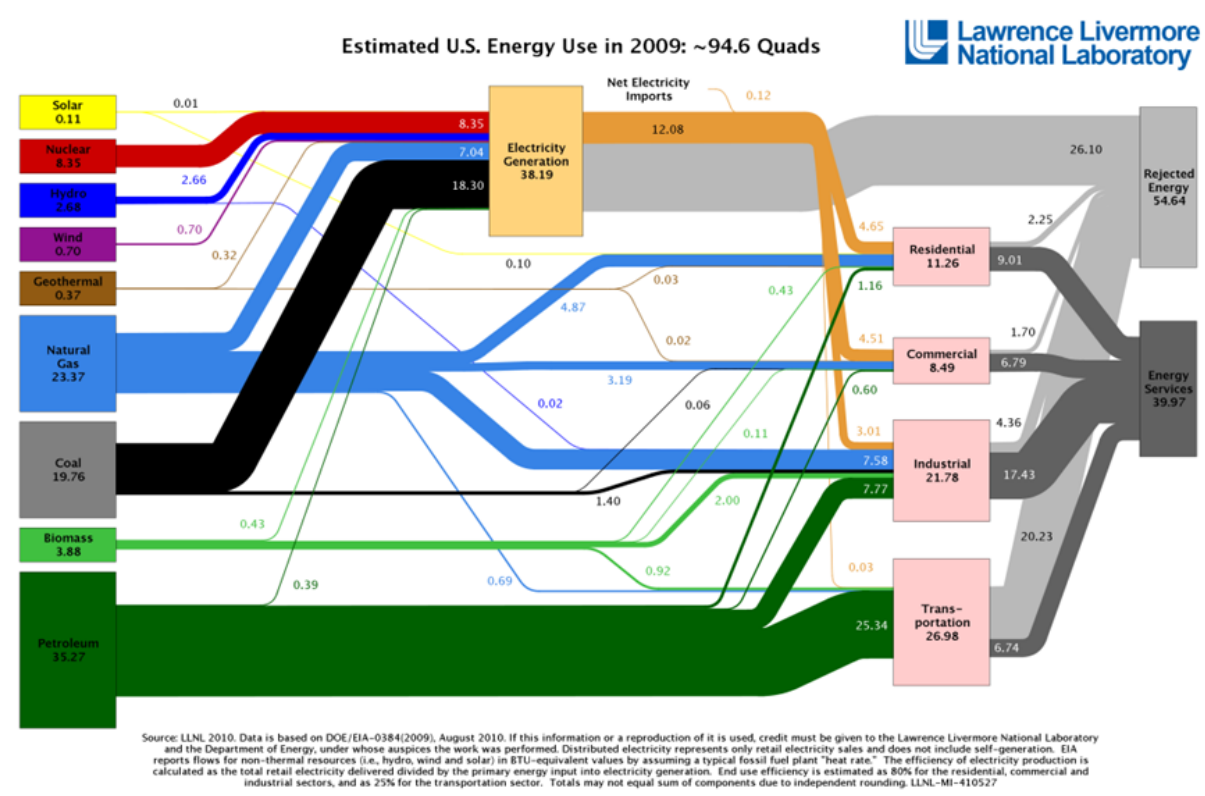

Figure 1: Primary sources, final consumption of energy and estimated energy use in 2009 in the USA. Electricity generation stands for a 38 percent of the total primary energy use in the country, and it is used in similar proportions in the residential, commercial and industrial sectors. Source: Lawrence Livermore National Laboratory, https://flowcharts.llnl.gov/energy.html, accessed July, 26 $6^{\text {th }}, 2013$.

Having a central role in modern human economies and considering the permanent and positive relationship of energy consumption with any form of economic growth (Smil, 2008), electricity consumption scaling with population can strongly constrain the particular dynamics involved in productivity and economic growth in cities. The relationship between urban size and productivity is indeed a central fact of urban economics (Glaeser and 
Resseger, 2010) but from a global environmental accounting point of view energy consumption can effectively restrain economic, social and environmental sustainability, and might counterbalance advantages coming from technological innovations and agglomeration economies (Carlino et al., 2007; Rauch, 1993).

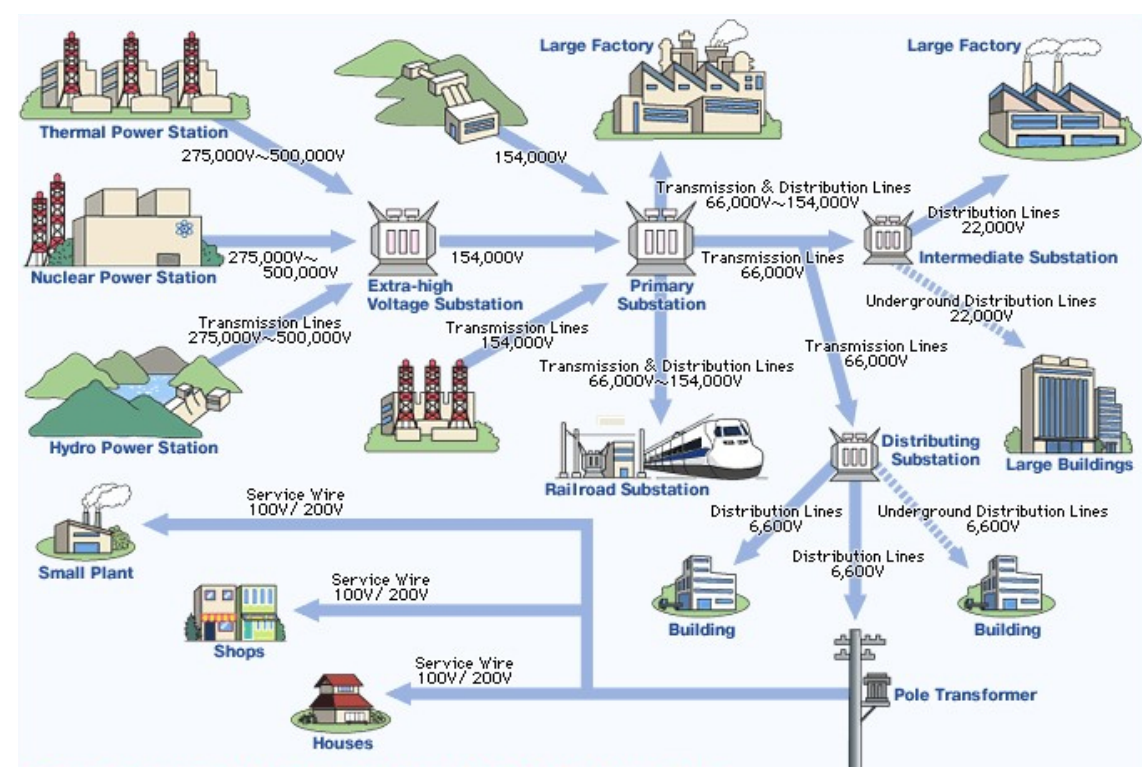

Figure 2: Process of generation, transmission, distribution and consumption of electrical energy. The voltage values may vary, depending on each country. Source: Tokyo Electric Power Company, http://www.tepco.co.jp/en/challenge/energy/pownet-e.html, accessed July, 26th, 2013.

In this particular case of energy related variables in cities, superlinear scaling exponents can be very significant. For example, while the required length of electrical conductors show an economy of scale with an exponent of 0.87, household electricity consumption is slightly superlinear, with an exponent of 1.04 (Table 1). Similar analysis performed in southern Spain and for different economic sectors shows even more variability (Table 2). Considering a 95\% confidence interval, total electricity consumption $(b=$ 1.05) and electricity consumption in each economic sector presents superlinear scaling exponents with population. Only primary and public sectors, with scaling exponent less than one, lie also within the linear and superlinear regimes when confidence intervals are taken into account (HortaBernús, 2010). Understanding how population growth can be related to superlinear scaling in energy consumption is an important objective to 
manage current urban (and global) challenges such as the effect of transport and industrial emissions on climate change, the use (and abuse) of natural resources or the obsolescence of energy infrastructures, to name just a few of them.

\begin{tabular}{lccc}
\hline \multicolumn{1}{c}{ Sector } & $\boldsymbol{b}$ & $\mathbf{9 5 \%} \mathbf{C I}$ & $\mathbf{R}^{\mathbf{2}}$ \\
\hline Primary & 0.95 & {$[0.86,1.04]$} & 0.72 \\
Secondary & 1.32 & {$[1.23,1.41]$} & 0.76 \\
Tertiary & 1.30 & {$[1.27,1.33]$} & 0.91 \\
Household & 1.07 & {$[1.06,1.08]$} & 0.96 \\
Public & 0.99 & {$[0.96,1.02]$} & 0.85 \\
All & 1.05 & {$[1.03,1.07]$} & 0.96 \\
\hline
\end{tabular}

Table 2: Scaling exponents with adjusted $\mathrm{R}^{2}$ and 95 percent confidence interval, for electricity consumption and economic sectors in 2008, in Andalusia.

\section{Scaling and Obsolescence}

An energy infrastructure involves a chain of interacting processes and systems, including resource extraction, generation and production, storage, transmission and transportation, marketing, distribution, consumption, and waste disposal. In this context, the development of models to forecast energy demand is a fundamental field in the electricity sector of any developed country. Such models must incorporate the economic, social, technological and environmental aspects, and provide insights into the effect of policy decisions concerning energy on the well-being of private citizens and society as a whole. The importance of the provision of electricity is our inability to store large amounts of this energy vector, which means that the electrical system must remain in balance, thus producing the amount of electricity that is consumed in real time. To accomplish this objective we must anticipate the needs that will exist in different time horizons: in the short-term, to meet the demand of the end user in real time, in the mid-term, to plan the maintenance of power plants and networks, and in the long-term, to evaluate the planning of new generation, transmission or distribution facilities.

Scaling laws can significantly modify engineering analysis of obsolescence in energy infrastructural systems like power grids. Several models and mathematical algorithms have been worked out within the last 
decades to predict electrical energy requirements in specific areas, with variables like the cost of the energy sources, electrical requirement records, GDP, annual average temperatures, etc. (Jebaraj and Iniyan, 2006). Lately, additional trends have also been considered like decentralization of energy production, energy efficiency, technological evolution or the integration of renewable energies. But when nonlinear correlations between consumption and population are present, even the most precise spatial load forecasting analysis can be inaccurate.

Although an analytical model that takes into account scaling laws to forecast the precise date in which an energy infrastructure (i.e., a transmission line in the case of electricity) capacity will be below the power requirements of the geographical area being supplied might include many sources of uncertainty, simplified approaches can also give sufficient accuracy as a first approximation (Horta-Bernús et al., 2013). In the next section, an approach of this kind is applied to assess the approximate end of life of a high voltage transmission line located in Andalusia, the southernmost autonomous region of Spain, the second largest $\left(87,268 \mathrm{~km}^{2}\right)$ and the first one in population $(8,449,985$ inhabitants in 2012).

\section{An Example from Spain}

In this section we perform a very simple load forecasting analysis to detect the effect of allometric scaling in the obsolescence of a high-voltage transmission line located in Andalusia. Although the geographical area supplied by a transmission line cannot be exactly determined, it is well approximated by the geographical area associated to its substations. Since substations associated to the line under study are almost exclusively those located in the urban agglomerations of the autonomous region, we include the whole territory of Andalusia in the study. For this very simple case study, we assume that the transmission system remains the same during the study timeframe (e.g. no new lines or substations are built or upgraded) and that the loading of the transmission line under study is linearly correlated with the demand. Although these are very strong assumptions, the purpose of this example is not to develop a thorough forecasting and overloading analysis of an electric system, but only to put forward the impact that scaling laws, taken in a simple way, may have on the performance of these systems. It is our contention that there is a need for additional research on these issues to correctly assess the real evolution of electric systems under this kind of assumptions. 
Electrical consumption and population data from year 2001 until year 2009 has been made publically available by the authors at Google Public Data Explorer ('Explore the Data' option at http://tinyurl.com/l9y9jyk) where a map of the southern part of the Iberian Peninsula can be used to locate cities and towns which have been analyzed (http://tinyurl.com/ljp3se2). This data can be retrieved from the Instituto de Estadística de Andalucía ${ }^{1}$. Characteristic data for towns and cities in Andalusia for year 2002 is shown in Table 3.

\begin{tabular}{|ll}
\hline Data & Value \\
\hline Number of towns (with data for all sectors) & 660 \\
\hline Population in the biggest city (Sevilla) & 704,114 inhabitants \\
\hline Number of towns under 1,000 inhabitants & 190 \\
\hline Number of towns between 1,000 and 10,000 inhabitants & 445 \\
\hline Number of towns between 10,000 and 100,000 inhabitants & 122 \\
\hline Number of towns between 100,000 and 1,000.000 inhabitants & 12 \\
\hline Total Minimum Annual Consumption & $59 \mathrm{MWh}$ \\
\hline Total Maximum Annual Consumption & $2,298.994 \mathrm{MWh}$ \\
\hline Total Average Annual Consumption per Capita & $1.52 \mathrm{MWh}$ \\
\hline
\end{tabular}

Table 3: Characteristic data for towns and cities in Andalusia in 2002.

Table 4 presents numerical values for total electricity consumption scaling exponents between years 2002 and 2008. Figure 3 shows the graphical representation of the allometric scaling for 587 Andalusian cities and their inhabitants for year 2008. For every year, the power law regression can be translated into an equation like $Y \sim M^{b}$ where $Y$ is the total electrical energy consumption (in MWh) and $M$ is the number of inhabitants per urban context. As we can see in Table 4, throughout this period of analysis, $b$ exponent for total consumption shows a steady decrease from 1.13 to 1.05 . This variability might be explained by the many upheavals of the recent economic crisis, but no significant statistical correlation has been found between $b$ and any other economic or social variable. Nonetheless, since this variability implies no substantial qualitative changes on the final results and the characteristic scaling exponent for year 2009, the last one with reliable data, equals to 1.07,

${ }^{1}$ http://www.juntadeandalucia.es/institutodeestadisticaycartografia/sima/index2.htm

(C) 2013 The Society of Urban Technology 
in order to be conservative coefficient $b$ is considered equal to 1.05 throughout the whole analyzed period. In any case, it indicates a superlinear behavior and an electric consumption that increases faster than urban inhabitants. Thus a transmission line projected with no consideration of allometric behavior in cities being supplied by this line may reach its maximum load capacity before the expected date.

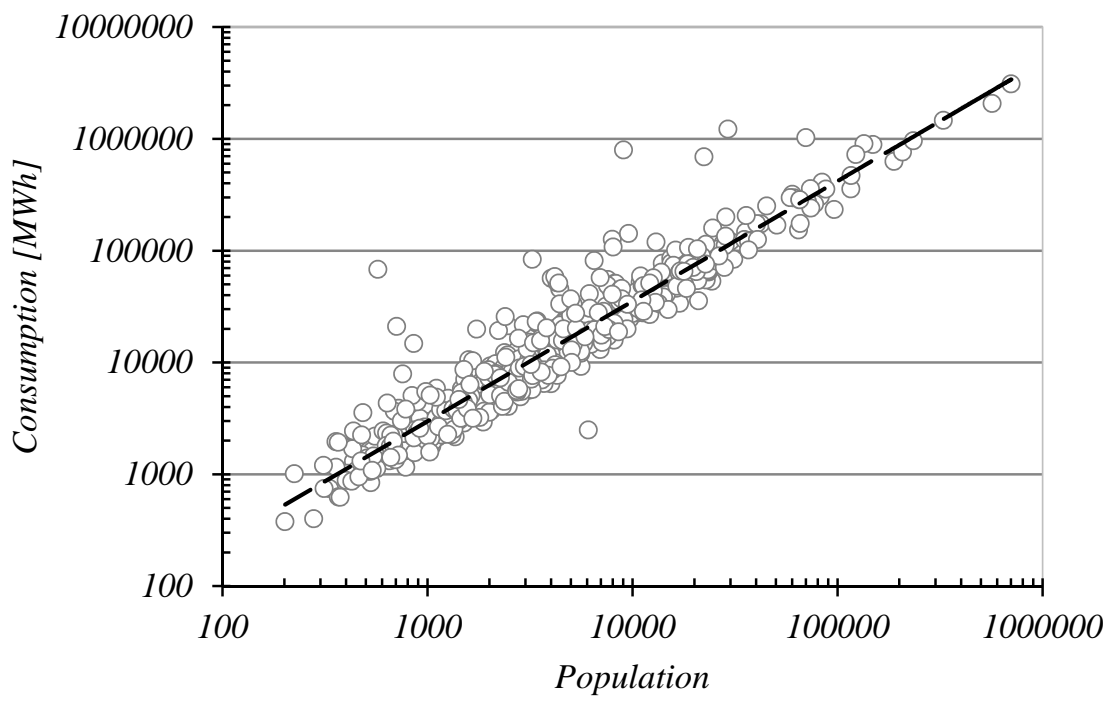

Figure 3: Slightly superlinear scaling of total electricity consumption for year 2008 in Andalusia autonomous region (Spain). This figure, plotted in double logarithmic axes, includes data for 587 cities and the long dashed line shows the best fit to a scaling relation $Y \sim M^{b}$, with $b=1.05\left(\mathrm{R}^{2}=0.90\right)$. (See Google Public Data Explorer, http://tinyurl.com/msa2wzx).

Electric energy and power are concepts typically used to define the electrical performance of a high-voltage system. Assuming that power is the instantaneous value of consumed energy in the course of time, a specific value of energy may have different power profiles. Here, it is assumed that load profiles (either daily, weekly, monthly or annual) do not suffer substantial fluctuations throughout periods (years) used for predictions.

\begin{tabular}{cccc}
\hline Year & $\boldsymbol{b}$ & $\mathbf{R}^{\mathbf{2}}$ & $\begin{array}{c}\text { Number of } \\
\text { cities included }\end{array}$ \\
\hline $\mathbf{2 0 0 2}$ & 1.13 & 0.87 & 660 \\
$\mathbf{2 0 0 4}$ & 1.11 & 0.88 & 565 \\
$\mathbf{2 0 0 6}$ & 1.10 & 0.89 & 581 \\
$\mathbf{2 0 0 8}$ & 1.05 & 0.90 & 587 \\
\hline
\end{tabular}


Table 4: Values for the total electric consumption scaling exponent $b$ in Andalusia and for different years.

Based on previous considerations, and assuming that population growth will inevitably occur among urban agglomerations (United Nations, 2012), any percentage increase in population will lead to a superlineal percentage increase in energy consumption. Population projections in Andalusia are shown in Table 5 under three different scenarios which correspond to three population growth projections for the autonomous region (S. Bermúdez and J. Planelles, 2011). All scenarios present nonlinear population growths, which can be well fitted by simple quadratic equations.

\begin{tabular}{l|ccc}
\hline \multirow{2}{*}{ Scenario } & $\mathbf{3}$ & Year \\
& $\mathbf{2 0 0 9}$ & $\mathbf{2 0 2 5}$ & $\mathbf{2 0 5 0}$ \\
\hline Low & 8.45 & 8.85 & 8.89 \\
Medium & 8.45 & 9.10 & 9.73 \\
High & 8.45 & 9.37 & 10.66 \\
\hline
\end{tabular}

Table 5: Population forecast for Andalusia (2009 - 2035). Numbers show inhabitants divided by $10^{6}$.

With these population projections, load forecasting results that take into account allometric patterns can be easily obtained: final consumption $Y$, and thus needed electric power, will be proportional to the $b$ power of projected population $M$. These results are shown in Table 6, where they are compared to a reference power value for each year (Ref. column). This reference value does not take into account allometric concepts and it has been constructed according to the energy forecast from year 2009 to 2035, which gives a 1.46 percent constant annual increase in electric energy consumption for the region (M. Valero, 2010). Variables considered in this forecast have been GDP per capita, housing stock, constant relative prices of electricity and oil, industrial productive capacity, and minimum and maximum annual temperatures. In order to properly scale the reference column, real values for the case study electrical line in the reference year 2009 have been considered (Ministerio de Política Territorial y Administración Pública, 2011). In this case power required in 2009 is 1,700 MW and the maximum (nominal) allowable power transmitted by the line is $2,400 \mathrm{MW}$, approximately 40 percent under that of 
the reference year value. Allometric (low, medium and high) scenarios consider the previous population settings (in cumulated percentage in Table 6) to illustrate the scaling possibilities of electrical energy requirements. With allometric considerations, the moment in time when demand exceeds nominal power capacity is brought forward by 3 years (low setting) and 7 years (high setting) in respect to the final reference year, 2033 (grey shaded cells in Table 6). Thus, the time span in which this case line may satisfy power requirements has been shortened by 12 percent to 30 percent respectively.

\begin{tabular}{c|c|cc|cc|cc}
\hline & Ref. & \multicolumn{2}{|c|}{ Low } & \multicolumn{2}{c|}{ Medium } & \multicolumn{2}{c}{ High } \\
Year & MW & \% & MW & \% & MW & \% & MW \\
\hline $\mathbf{2 0 0 9}$ (ref.) & 1,700 & & 1,700 & & 1,700 & & 1,700 \\
$\mathbf{2 0 1 0}$ & 1,725 & 0.46 & 1,733 & 0.58 & 1,735 & 0.81 & 1,739 \\
$\mathbf{2 0 1 2}$ & 1,776 & 1.24 & 1,798 & 1.75 & 1,807 & 2.31 & 1,817 \\
\hline $\mathbf{2 0 1 6}$ & 1,882 & 2.66 & 1,932 & 3.77 & 1,953 & 5.05 & 1,977 \\
$\mathbf{2 0 2 2}$ & 2,052 & 4.09 & 2,136 & 6.24 & 2,180 & 8.63 & 2,229 \\
\hline $\mathbf{2 0 2 6}$ & 2,175 & 4.64 & 2,276 & 7.45 & 2,337 & 10.53 & 2,404 \\
\hline $\mathbf{2 0 2 8}$ & 2,239 & 4.87 & 2,348 & 7.99 & 2,418 & 11.39 & 2,494 \\
$\mathbf{2 0 3 0}$ & 2,305 & 4.99 & 2,420 & 8.42 & 2,499 & 12.23 & 2,587 \\
$\mathbf{2 0 3 2}$ & 2,373 & 5.06 & 2,493 & 8.85 & 2,583 & 12.94 & 2,680 \\
\cline { 2 - 7 } $\mathbf{2 0 3 3}$ & 2,407 & 5.11 & 2,530 & 9.06 & 2,625 & 13.34 & 2,728 \\
\hline
\end{tabular}

Table 6: Scenarios and power projections for the real transmission line selected as a case study. Percentages shown are the final result considering both increments, nonlinear population growth and superlinear (i.e., $b=1.05$ ) consumption scaling with population. Nominal (maximum) power is set at 2,400 MW.

\section{Discussion and Conclusions}

Cities accommodate the spatial location and impulse for most of the planet's innovation, ingenuity and vision. They are complex systems and follow complex dynamical processes which our perception organizes according to particular spatial and temporal patterns. In spite of each city's distinctive complexity, when urban agglomerations are taken as a whole, some kind of orderliness appears in the form of scaling correlations. These scaling laws are also present in biology and ecology, where economies of scale are the usual outcome. Defined as in microeconomics, economies of scale are the cost advantages that systems obtain due to size, with cost per unit of output generally decreasing with increasing scale. But unlike living organisms, urban 
agglomerations present much more variability: economies of scale are present but also the opposite behavior exists, that is the output increase by more than that proportional change. While the former are related to infrastructural events (i.e., service stations, road surface, electric cables, etc.), this latter kind of behavior, known as increasing returns, tends to be related with social, economic, and cultural interactions (i.e., patents, inventions, researchers, gross domestic product, etc.) and it usually leads to higher productivity and innovation. This pattern comes obviously not for free and this cost is commonly, and ultimately, transmitted to the energy sectors (essentially secondary, tertiary and household sectors). Scaling relations for these categories are clearly superlinear, which means that increasing returns are at play.

In the particular case of electricity, this nonlinear statistical trait might remarkably constrain the lifetime of their transmissions and distribution infrastructures and accelerate the unsustainability of a city's energy sphere. Since by mid-century, almost two thirds of the world's population will be urban dwellers, this aggregate superlinear energetic behavior must be taken into account if we want to avoid any kind of urban collapse. The very simple example accounted for in this paper shows a substantial reduction in the lifespan of a transmission line when this laws are considered. Thus, incorporating this kind of consumption patterns suggested by scaling laws can help in better anticipating the point in time when electricity infrastructures may be insufficient to feed consumer demand. Results presented in this paper, albeit following strong assumptions, might be useful in predicting the estimated year in which the transmission capacity of a line in project phase will be exceeded.

\section{Emerging Challenges for Cities and the Future Global Energy System}

Even though the universality of scaling laws in urban contexts is still under discussion, they are nonetheless the ultimate patterns of cities' aggregate behavior. Superlinear scaling in energy issues ultimately implies unsustainable socioeconomic processes which may lead to the collapse of the energy system of a region, or even a country, and make cities increasingly unsecure and disturbing places to live. If scaling laws seem an inevitable emergent feature of these systems, what implications have the present urban world population growth trends and demand for energy in the reconfiguration of urban energy systems? 
Although the answers to this question are numerous, and they should primarily include a deliberate and conscious individual reduction in energy consumption of any kind, most of them will essentially depend on technological and regulatory innovations (Dodgson and Gann, 2010). These should help us developing less vulnerable energy systems using explicit tools, methodologies and science-policy integrated processes to better represent and assess local energy systems dynamics, and to incorporate the social, political and behavioral dimensions in the modeling of these same systems from a global point of view.

In the present globally interconnected world, energy is generated, stored, transmitted and consumed (and its waste disposed or, luckily, recycled) through a complex and dynamic system of systems. Thus, a cardinal challenge is to focus on the multiple interactions of different scales: not only in its operational size and interconnectedness, but also in its other non-energy subsystems, like information technologies, governance, financial, urban design, etc. Radical changes in the global energy system will require coupling the synergies of innovations occurring within the energy systems at various scales with those being developed out of them. But how this ambitious objective can be achieved? The smart-grid concept seems to be an obvious choice. Connecting it with the smart-cities development (Dirks and Keeling, 2009), it may represent a full suite of answers to complex problems that concern the sustainability and optimality of energy, information and transport systems in urban contexts. But together with these two currently widespread concepts, there are many other options that must be explored. These include the individual motives, incentives and learning capabilities of the agents constituting the global system, together with their interactions and under an evolutionary perspective (Solé et al., 2013).

In the time to come, a globally smart energy system, coupled with other systems, can only be composed by 'energy-intelligent agents' who know what is best needed to be done for optimizing their multiple decisions in ways that lead to a secure, sustainable and high quality operational global energy system. Adequate models, simulations, and large data management tools will be needed to capture the global (spatial and temporal) dimension of energy and electricity systems, with as much reduced uncertainty as possible. This is not an easy task. To assess uncertainty of model-based prediction, and in particular with regard to generating a better understanding of systems of cities and global energy system dynamics, we need to understand sensitivities and to identify which model parameters are most important to understand what drives a system's behavior. This can only be achieved by taking a complex perspective, able to deal with non-linear behaviors, bifurcations, tipping 
points, complex networks and rapid changes in boundary conditions. Only with this kind of global analysis and complexity science approach it will be possible to properly address the economic, environmental, and social consequences of our choices concerning technological development in urban contexts.

\section{Acknowledgements}

The authors wish to specially thank Marilà Lucas-Serra for most helpful conversations, support and suggestions.

\section{Note on Contributors}

Ricard Horta-Bernús and Martí Rosas-Casals are associate professors at the Terrassa Engineering School (EET) at the Universitat Politècnica de Catalunya - Barcelona Tech (Barcelona, Spain). Both of them are research staff at the Sustainability Measurement and Modeling Laboratory (SUMM Lab) of the same school.

\section{Bibliography}

E. Arcaute, E. Hatna, P. Ferguson, H. Youn, A. Johansson and Michael Batty, "City Boundaries and the Universality of Scaling Laws," arXiv:1301.1674v1 [physics.soc-ph] (2013)

M. Batty, Cities and Complexity: Understanding Cities with Cellular Automata, Agent-Based Models, and Fractals (Cambridge, MA: MIT Press, 2007).

M. Batty and P. Longley, Fractal Cities: A Geometry of Form and Function (London, UK: Academic Press, 1994).

S. Bermúdez and J. Planelles, "Proyección de población activa de Andalucía 2009 - 2035," Documentos de Trabajo del Instituto de Estadística de Andalucía, (Sevilla: EIA, 2011). 
L.M.A. Bettencourt, J. Lobo, D. Helbing, C. Kühnert and G.B. West, "Growth, innovation, scaling and the pace of life in cities," Proceedings of the National Academy of Sciences of the USA, 104 (2007) 7301-7306.

L.M.A. Bettencourt and G.B. West, "A unified theory of urban living," Nature, 467 (2010) 912-913.

L.M.A. Bettencourt, J. Lobo, D. Strumsky and G.B. West, "Urban scaling and its deviations: revealing the structure of wealth, innovation and crime across cities,” Public Library of Science ONE, 5:11 (2010) e13541

L.M.A. Bettencourt, “The Origins of Scaling in Cities,” Science 340 (2013) 1438-1441.

G.A. Carlino, S. Chatterjee and R.M. Hunt, "Urban density and the rate of invention,” Journal of Urban Economics 61 (2007) 389-419.

D. S. Dendrinos and H. Mullally, Urban Evolution: Studies in the Mathematical Ecology of Cities (Oxford: Oxford Univ. Press, 1985).

S. Dirks and M. Keeling, A Vision of Smarter Cities: How Cities Can Lead the Way Into a Prosperous and Sustainable Future (New York: IBM Institute for Business Value, 2009).

M. Dodgson and D. Gann, Innovation: A Very Short Introduction (Oxford: Oxford University Press, 2010).

M. Dodgson and D. Gann, "Technological Innovation and Complex Systems in Cities,” The Journal of Urban Technology 18:3 (2011) 101-113.

E.L. Glaeser and M.G. Resseger, "The Complementarity Between Cities and Skills,” Journal of Regional Science, 50 (2010) 221-244.

R. Horta-Bernús, M. Rosas-Casals and S. Valverde, "Discerning Electricity Consumption Patterns from Urban Allometric Scaling," paper presented at Complexity in Engineering 2010 (Rome, 22-24 Feb. 2010). DOI: 10.1109/COMPENG.2010.29 
R. Horta-Bernús and M. Rosas-Casals, "Modification of the Perrine-Baum Diagram to Improve the Calculation of High Voltage Transmission Lines," IEEE Transactions on Education, 56:3 (2013) 274-279.

S. Jebaraj and S. Iniyan, "A review of energy models," Renewable and Sustainable Energy Review 10 (2006) 281-311.

M. Kleiber, “Body Size and Metabolism,” Hilgardia 6 (1932) 315-351.

C.D. Kühnert, D. Helbing and G.B. West, "Scaling laws in urban supply networks," Physica A: Statistical Mechanics and its Applications 363:1 (2006) 96-103.

S. Lämmer, B. Gehlsen and D. Helbing, "Scaling laws in the spatial structure of urban road networks," Physica A: Statistical Mechanics and its Applications 363:1 (2006) 89 - 95.

J.D. Marshall, "Urban land area and population growth: a new scaling relationship for metropolitan expansion,” Urban Studies, 44:10 (2007) 18891904.

Ministerio de Política Territorial y Administración Pública, Boletín Oficial del Estado (BOE), $n^{\circ} 142$, pp. 67702-67703 (Madrid, Spain: Ministerio de Política Territorial y Administración Pública, 2011).

L. Mumford, The City in History: Its Origins, Its Transformations, and Its Prospects (New York: Harcourt, Brace \& World, Inc., 1961).

M.E. Nye, When the Lights Went Out. A History of Blackouts in America (Cambridge MA: MIT Press, 2010).

J.E. Rauch, "Productivity gains from geographic concentration of human capital: evidence from cities,” Journal of Urban Economics 34 (1993) 380400 .

H.D. Rozenfeld, D. Rybski, J.S. Andrade, M. Batty, H.E. Stanley and H.A. Makse, "Laws of Population Growth," Proceedings of the National Academy of Sciences of the USA, 105 (2008) 18702-18707.

(C) 2013 The Society of Urban Technology 
R. Solé, S. Valverde, M. Rosas-Casals, S. Kauffman, D. Farmer, and N. Eldredge, "The Evolutionary Ecology of Technological Innovations," Complexity 18:4 (2013) 15-27.

V. Smil, Energy in Nature and Society: General Energetics of Complex Systems (Cambridge MA: MIT Press, 2008).

United Nations, World urbanization Prospects: the 2011 Revision (New York: United Nations, 2012).

M. Valero, "La demanda de electricidad en España. Previsión 2035,” Boletín Económico de Información Comercial Española, 2987 (2010) 23-32.

G.B. West, J. Brown, and B. Enquist, "A general model for the origin of allometric scaling laws in biology,” Science, 276 (1997) 122-126.

G.B. West and J. Brown, "The Origin of Allometric Scaling Laws in Biology from Genomes to Ecosystems: Towards a Quantitative Unifying Theory of Biological Structure and Organization,” Journal of Experimental Biology, 208 (2005) 1575-1592.

G.K. Zipf, Human Behavior and the Principle of Least Effort (Cambridge, MA: Addison-Wesley, 1949) 